\title{
ON A NONLINEAR REACTION-DIFFUSION BOUNDARY-VALUE PROBLEM: APPLICATION OF A LIE-BÄCKLUND SYMMETRY
}

\author{
PHILIP BROADBRIDGE ${ }^{1}$ and COLIN ROGERS ${ }^{2}$
}

(Received 5 April 1991; revised 24 October 1991)

\begin{abstract}
By a systematic search for Lie-Bäcklund symmetries, a class of linearisable reactiondiffusion equations is obtained that has, as a canonical form, $u_{t}=u^{2} u_{x x}+2 u^{2}$. One such nonlinear equation is

$$
\theta_{t}=\partial_{x}\left[a(b-\theta)^{-2} \theta_{x}\right]-m a(b-\theta)^{-2} \theta_{x}-q \exp (-m x) .
$$

This represents an extension of Fokas-Yortsos-Rosen equation $(q=0)$ to incorporate a reaction term. It is relevant to the modelling of unsaturated flow in a soil with a volumetric extraction mechanism, such as a web of plant roots. Here, a reciprocal transformation is used to solve a nonlinear boundary-value problem for transient flow into a finite layer of a soil subject to a constant flux boundary condition to compensate for such water extraction.
\end{abstract}

\section{Introduction}

Nonlinear reaction-diffusion equations of the type

$$
\partial_{t} \theta=\partial_{x}\left[D(\theta) \theta_{x}-K(\theta)\right]+Q(x, t, \theta)
$$

arise in a number of physical applications such as chemical reaction modelling and population dynamics $[18,2]$. In the present context of the modelling of water transport through unsaturated soils, $\theta$ represents liquid concentration, while a negative reaction term $Q$ represents a rate of extraction. In the model of [28] this extraction is due to a web of plant roots. The nonlinearity of the diffusivity $D(\theta)$ is an important factor in determining the concentration profile [21].

\footnotetext{
${ }^{1}$ Department of Mathematics, University of Wollongong, Wollongong, NSW.

${ }^{2}$ Department of Mathematical Sciences, Loughborough University of Technology, U.K.

(C) Copyright Australian Mathematical Society 1992, Serial-fee code 0334-2700/92
} 
Under the Kirchhoff transformation

$$
\mu=\int_{\theta_{0}}^{\theta} D(\sigma) d \sigma
$$

where $\theta_{0}$ is an appropriate constant, (1.1) adopts the alternative form

$$
\partial_{t} \mu=\Delta(\mu) \mu_{x x}+\Sigma(\mu) \mu_{x}+R(x, t, \mu)
$$

where $R \equiv D Q$. The admittance of Lie-Bäcklund symmetries was used in [9] as a basis to claim that the most general nonlinear equation of the type

$$
\partial_{t} \mu=\Delta(\mu) \mu_{x x}+R\left(\mu, \mu_{x}\right)
$$

which is linearisable is

$$
\partial_{t} \mu=\Delta \mu_{x x}+\left[\Delta \Delta^{\prime \prime} / \Delta^{\prime}-\Delta^{\prime} / 2\right] \mu_{x}^{2}+\alpha \Delta \mu_{x}, \quad \Delta^{\prime} \neq 0,
$$

where $\Delta=\Delta(\mu)$ has arbitrary dependence of $\mu$ and $\alpha$ is an arbitrary constant. In terms of a new dependent variable $\bar{\mu}=\Delta(\mu)^{1 / 2}$, (1.5) reduces to

$$
\partial_{t} \bar{\mu}=\bar{\mu}^{2} \bar{\mu}_{x x}+\alpha \bar{\mu}^{2} \bar{\mu}_{x},
$$

or, if we set $\bar{\theta} \equiv \bar{\mu}^{-1}$,

$$
\partial_{t} \bar{\theta}=\partial_{x}\left[\bar{\theta}^{-2} \bar{\theta}_{x}\right]+\alpha \bar{\theta}^{-2} \bar{\theta}_{x} .
$$

However, in a recent development, Freeman and Satsuma [10] showed that the nonlinear equation

$$
\partial_{t} \rho=\rho^{2} \rho_{x x}+2 \rho^{2}
$$

in linearisable via a reciprocal-type transformation. An explicit pulse solution with compact support along with an exact solution descriptive of interaction between pulses was thereby derived. The result in [10] appears to contradict that of Fokas and Yortsos in [9] since the nonlinear equation (1.8) is not included in the class (1.6) although the latter does include an important nonlinear convective term. If invariance under a broader class of Lie-Bäcklund transformations than that in [9] is investigated, an associated wider class to linearisable equations is derived $[11,31]$. Moreover, it incorporates equation (1.8) as a canonical reduction. The latter admits a third-order Lie-Bäcklund symmetry with explicit dependence on the $x$-variable. This resolution lends support to the Lie-Bäcklund approach to linearisation $[1,3,5,14]$.

In order for a nonlinear partial differential equation to be linearisable, it must possess Lie-Bäcklund symmetries of arbitrarily high order. Any secondorder nonlinear evolution equation

$$
\theta_{t}=F\left(x, \theta, \theta_{x}, \theta_{x x}\right)
$$


that possesses a Lie-Bäcklund symmetry of order $n \geq 3$ can be reduced by a contact transformation, to one of the canonical forms $[11,31]$ :

$$
\begin{aligned}
& \theta_{t}=\theta_{x x}+g(x) \theta, \\
& \theta_{t}=\theta_{x x}+2 \theta \theta_{x}+g^{\prime}(x), \\
& \theta_{t}=\partial_{x}\left[\theta^{-2} \theta_{x}\right]+c_{1}\left[\theta+x \theta_{x}\right]+c_{2} \theta_{x}, \\
& \theta_{t}=\partial_{x}\left[\theta^{-2} \theta_{x}\right]-2 .
\end{aligned}
$$

For each of these, Galaktionov et al. [11] give a recursion operator that can generate a chain of infinitesimal Lie-Bäcklund symmetries of arbitrarily high order. Each of the above equations can be transformed to a linear equation but to achieve this, in some cases, a potential variable must be introduced.

In our hydrological application, we have a sink term that depends on position $x$. Hence, we require the sub-class of equations (1.1), including those with $x$-dependent sink term, that can be transformed to one of the above canonical forms. The full equivalence classes, containing the above canonical forms, have not been published explicitly, although it seems that they may have been given in unpublished work by Svirshchevskii, cited by Galaktionov et al. [11]. By carrying out a Lie-Bäcklund symmetry analysis, we explicitly characterise the full class of equations (1.1) that can be linearised. Furthermore we exploit this class of equations to solve a practical nonlinear boundary-value problem.

The linearisation procedure in the present paper involves the use of reciprocal transformations. The latter have been used in the recent past to solve a number of physically important nonlinear boundary-value problems, notably involving heat conduction and oil/water transport through soils [2226]. Their application to nonlinear integrable equations of soliton theory in linking inverse scattering schemes and generating auto-Bäcklund transformations is recorded in $[16,27]$. Here, a reciprocal transformation is used to solve a nonlinear boundary-value problem which models the transport of water through a bounded soil with surface infiltration and an interior extraction mechanism.

\section{A new class of linearisablea reaction-diffusion equations}

The symmetry analysis of Fokas and Yortsos [9] may, in fact, be extended to allow non-autonomous infinitesimal symmetries

$$
\mu^{*}=\mu+s L\left(x, t, \mu, \mu_{1}, \mu_{2}, \mu_{3}\right)+O\left(s^{2}\right)
$$


where $L$ has explicit dependence on $x$ and $t$ as well as on

$$
\mu_{j} \equiv\left(\partial / \partial_{x}\right)^{j} \mu(x, t)
$$

$j=0,1,2,3^{3}$. The requirement that the nonlinear equation

$$
\partial_{t} \mu=\Delta(\mu) \mu_{x x}+R(x, t, \mu)
$$

be invariant under the class of symmetries (2.1) leads to a set of determining equations for $L$. These comprise a system of linear partial differential equations for $L$ which have no solution unless $\Delta(\mu)$ and $R(x, t, \mu)$ satisfy certain consistency requirements. In the Appendix, these consistency conditions are derived and solved. It is there shown that (2.2) admits a symmetry of the type (2.1) if and only if it adopts the form

$$
\partial_{t} \mu=(\alpha \mu+\beta)^{2} \mu_{x x}+(\alpha \mu+\beta)^{2} f(t) / \alpha^{2}+(\alpha \mu+\beta) f^{\prime}(t) /(\alpha f(t))
$$

or, equivalently, with $b-w \equiv a^{1 / 2}(\alpha \mu+\beta)^{-1}, a=\alpha^{2} \neq 0$,

$$
\partial_{t} w=\partial_{x}\left[a(b-w)^{-2} w_{x}\right]+(b-w) f^{\prime}(t) / f(t)+f(t),
$$

with $f(t)$ an arbitrary differentiable function and $a, b$ arbitrary constants.

In terms of new dependent and independent variables $\rho, \xi, \tau$ introduced according to

$$
\begin{gathered}
\rho=2 \alpha(\alpha \mu+\beta) / f(t)=2 a /(b-w) f(t), \\
\xi=x, \quad \tau=\frac{1}{4 a} \int_{t_{0}}^{t} f^{2}(T) d T,
\end{gathered}
$$

it is seen that (2.3) and (2.4) adopt precisely the canonical form

$$
\partial_{\tau} \rho=\rho^{2} \rho_{\xi \xi}+2 \rho^{2} .
$$

However, so far (2.4) lacks a nonlinear convective term which, in the context of hydrology, represents the action of gravity, an important agent of transport in the late stages of infiltration [21]. In order to incorporate a convective term, we boost the above result by applying a transformation previously used in the case of reaction-free diffusion, namely [4]

$$
x=m^{-1}[1-\exp (-m z)], \quad b-w=(b-\theta) \exp (m z)
$$

where $m \neq 0$, so that $(2.4)$ becomes

$$
\partial_{t} \theta=\partial_{z}\left[a(b-\theta)^{-2} \theta_{z}\right]-m a(b-\theta)^{-2} \theta_{z}+f(t) e^{-m z}+(b-\theta) f^{\prime}(t) / f(t) .
$$

\footnotetext{
${ }^{3}$ In restricting $L$ to be of the third order, we are guided by the work of Bluman and Kumei [1] for the case $R=0$ wherein no new results were obtained by proceeding to higher orders.
} 
The latter represents an extension of the integrable Fokas-Yortsos-Rosen nonlinear diffusion-convection equation $[9,29]$ to incorporate an additional reaction term.

The nonlinear diffusivity of the form $D(\theta)=a(b-\theta)^{-2}$, appearing in (2.7), leads to useful exact solutions in the theory of unsaturated flow $[5,15$, 30]. The position of the singularity $\theta=b$ is taken just above the saturated water context $\theta_{s}$, so that $D(\theta)$ increases strongly near $\theta=\theta_{s}$ as commonly observed in laboratory experiments [34].

Not only has the nonlinear convection term now been included in (2.7), but the reaction term can now depend on position. This is a highly desirable feature in, for instance, plant root extraction models. A nonlinear boundary value problem relevant to such an application is solved via a reciprocal transformation in the next section. It illustrates the application of reciprocal transformations to the linearisation of the present class of nonlinear equations which admit Lie-Bäcklund symmetries.

\section{Exact solution of a nonlinear model for unsaturated flow in a soil with volumetric extraction}

The nonlinear equation

$$
\partial_{t} \theta=\partial_{z}\left[D(\theta) \theta_{z}\right]-K^{\prime}(\theta) \theta_{z}-Q(t, z, \theta)
$$

has been proposed as a macroscopic, one-dimensional model for vertical unsaturated flow in a soil with extraction by plant roots. In that context, $\theta$ is the volumetric water content (fraction of total soil volume occupied by water), $D$ is the soil/water diffusivity, $K$ is the hydraulic conductivity and $Q$ is the volumetric extraction rate per unit total soil volume: $z$ indicates depth beneath the soil surface and $t$ the time. $K(\theta)$ is increasing and concave as is $D(\theta)$ provided $\theta$ is large enough so that transport in the vapour phase is negligible [21]. The extraction term $Q$ is averaged over a horizontal soil cross-section containing a number of plant roots. The $z$-dependence of $Q$ is retained, as the plant root density must diminish at some depth.

Lomen and Warrick [17] have solved linear water-extraction models with $D(\theta)$ and $K^{\prime}(\theta)$ constant. However, so far, solutions to boundary-value problems involving the nonlinear equation (3.1) have only been obtained numerically [19]. By contrast, here, we construct the analytic solution of a boundary-value problem for a special nonlinear model namely

$$
\partial_{t} \theta=\partial_{z}\left[D(\theta) \theta_{z}\right]-K^{\prime}(\theta) \theta_{z}+Q(z)
$$

with

$$
D(\theta)=a(b-\theta)^{-2}, \quad K(\theta)=m a(b-\theta)^{-1}, \quad Q(z)=-q \exp (-m z),
$$


where $a, b, m$ and $q$ are constants available for approximation purposes. It is noted that in (3.3), the extraction term depends only on depth. In fact, in many situations, the dependence of $Q$ on $\theta$ and $t$ is not predominant. Thus, for example, it has been observed that some eucalypt species continue to draw steadily from unsaturated soil even throughout a drought [33]. Here, we make the simplifying assumption that the plants continue to extract water at a constant rate, provided the water content exceeds some critical value $\theta_{n}$ near wilting point. The range of water content considered is $\theta_{n}<\theta \leq \theta_{s}$ where $\theta_{s}$ is the water content at saturation. In the special model (3.2)-(3.3), the plant root length scale $m^{-1}$ is identical to the sorptive length scale $\ell_{s}$. The latter may be regarded as a representative capillary rise [35].

$$
\ell_{s}=\int_{\theta_{n}}^{\theta_{s}} D(\theta) /\left[K\left(\theta_{s}\right)-K\left(\theta_{n}\right)\right] d \theta=m^{-1}
$$

on use of (3.3). In practice, the sorptive length is often in the range $20-80 \mathrm{~cm}$ [34], within the same order of magnitude as plant root depths. Our assumed equality of $\mathrm{m}^{-1}$ and $\ell_{s}$ is restrictive but not unrealistic.

We assume that water is supplied at the surface $z=0$ at a rate $\bar{R}$ units of depth per unit time, to compensate for the water lost by extraction over the entire layer of depth $\ell$. The constant-flux boundary condition is

$$
m a(b-\theta)^{-1}-a(b-\theta)^{-2} \theta_{z}=\bar{R} \quad \text { at } z=0, t>0,
$$

where

$$
\bar{R}=\int_{0}^{\ell}-Q(z) d z=\frac{q}{m}[1-\exp (-m \ell)] .
$$

Further, it is assumed that the soil layer has an impermeable basement at $z=\ell$. The corresponding zero-flux boundary condition is

$$
m a(b-\theta)^{-1}-a(b-\theta)^{-2} \theta_{z}=0 \quad \text { at } z=\ell, t>0 .
$$

The initial condition corresponds to some prescribed water content profile

$$
\theta=\theta_{0}(z) \quad \text { at } t=0,0 \leq z \leq \ell .
$$

New variables $x$ and $w$ are now introduced as in (2.6 a,b) whence (3.2)(3.3) yields

$$
\partial_{t} w=\partial_{x}\left[a(b-w)^{-2} w_{x}\right]-q
$$

while the boundary and initial conditions become

$$
\begin{aligned}
-a(b-w)^{-2} w_{x} & =\bar{R} \quad \text { at } x=0, \\
w_{x} & =0 \quad \text { at } x=x_{1}:=m^{-1}[1-\exp (-m \ell)], \\
w(x, 0)-b & =\left\{\theta_{0}\left(-m^{-1} \ln [1-m x], 0\right)-b\right\} /\{1-m x\}
\end{aligned}
$$


If we further set

$$
\begin{gathered}
\rho=\frac{-2 a}{q(b-w)}, \\
s=\frac{q^{2} t}{4 a}
\end{gathered}
$$

then the boundary-value problem to be solved reduces to

$$
\begin{aligned}
\partial_{s} \rho & =\rho^{2} \rho_{x x}+2 \rho^{2}, \quad 0<x<x_{1}, \quad s>0, \\
\rho_{x} & =R^{*} \quad \text { at } x=0, s>0, \\
\rho_{x} & =0 \quad \text { at } x=x_{1}, s>0, \\
\rho & =\rho_{0}(x) \quad \text { at } s=0,0 \leq x \leq x_{1},
\end{aligned}
$$

where

$$
\begin{aligned}
R^{*} & =\frac{2}{q} \bar{R}=2 x_{1}, \\
\rho_{0}(x) & =\frac{2 a}{q} \frac{1-m x}{\left\{\theta_{0}\left(-m^{-1} \ln [1-m x], 0\right)-b\right\}} .
\end{aligned}
$$

Alternatively, in terms of the variables

$$
u=x-x_{1}, \quad \Psi=\rho^{-1},
$$

the boundary-value problem (3.14)-(3.17) becomes

$$
\begin{gathered}
\partial_{s} \Psi=\partial_{u}\left[\frac{\Psi_{u}}{\Psi^{2}}-2 u\right], \quad-x_{1}<u<0, \\
\frac{-1}{\Psi^{2}} \Psi_{u}=2 x_{1} \quad \text { at } u=-x_{1}, s>0, \\
\Psi_{u}=0 \quad \text { at } u=0, s>0, \\
\rho=\rho_{0}\left(u+x_{1}\right) \quad \text { at } s=0,-x_{1} \leq u \leq 0 .
\end{gathered}
$$

Introduction of the reciprocal transformation ${ }^{4}$

$$
\mathscr{R}: d y=\Psi d u+\left(\frac{\Psi u}{\Psi^{2}}-2 u\right) d s, \quad \tau=s
$$

yields

$$
\begin{aligned}
& \frac{\partial y}{\partial u}=\Psi \\
& \frac{\partial y}{\partial s}=\int_{0}^{u} \frac{\partial}{\partial u}\left(\frac{\Psi_{u}}{\Psi^{2}}-2 u\right) d u+\left.\left(\frac{\Psi_{u}}{\Psi^{2}}-2 u\right)\right|_{u=0}=\frac{\partial}{\partial s} \int_{0}^{u} \Psi d u
\end{aligned}
$$

${ }^{4}$ Alternatively, a procedure analogous to that of Storm [32] may be employed. 
so that

$$
y=\int_{0}^{u} \Psi d u=\int_{x_{1}}^{x} \rho^{-1}\left(x_{2}, x\right) d x_{2} .
$$

Reciprocally, (3.22) yields

$$
d u=\Psi^{-1} d y-\left(\frac{\Psi_{u}}{\Psi^{3}}-\frac{2 u}{\Psi}\right) d s=\rho d y+\left[\rho_{y}+2 \rho u\right] d \tau
$$

whence,

$$
\begin{aligned}
& \frac{\partial u}{\partial y}=\rho \\
& \frac{\partial u}{\partial \tau}=\int_{0}^{y} \frac{\partial}{\partial y}\left(\rho_{y}+2 \rho u\right) d y+\left.\left(\rho_{y}+2 \rho u\right)\right|_{y=0}=\frac{\partial}{\partial \tau} \int_{0}^{y} \rho d y
\end{aligned}
$$

so that

$$
u=\int_{0}^{y} \rho\left(y_{2}, s\right) d y_{2}=x-x_{1}
$$

Under $\mathscr{R}$,

$$
\partial_{s} \Psi=\partial_{u}\left[\frac{\Psi_{u}}{\Psi^{2}}-2 u\right] \leftrightarrow \partial_{\tau} \rho=\partial_{y}\left[\rho_{y}+2 \rho u\right]
$$

so that

$$
\int_{0}^{y} \rho_{\tau} d y=\rho_{y}+2 \rho u-\left.\left(\rho_{y}+2 \rho u\right)\right|_{y=0}=\rho_{y}+2 \rho u,
$$

and, on use of the reciprocal relation (3.24), we obtain Burgers' equation

$$
u_{\tau}=u_{y y}+2 u u_{y} \text {. }
$$

The upper boundary condition becomes

$$
u=-x_{1} \quad \text { at } y=\int_{x_{1}}^{0} \rho^{-1}\left(x_{2}, s\right) d x_{2} \text {. }
$$

But,

$$
\int_{x_{1}}^{0} \frac{\partial}{\partial x}\left[\rho_{x}+2 x\right] d x=\left.\left(\rho_{x}+2 x\right)\right|_{x=x_{1}} ^{x=0}=R^{*}-2 x_{1}=0,
$$

whence, on use of (3.14),

$$
\frac{\partial}{\partial s} \int_{x_{1}}^{0} \rho^{-1}\left(x_{2}, s\right) d x_{2}=0
$$

so that

$$
\int_{x_{1}}^{0} \rho^{-1}\left(x_{2}, s\right) d x_{2}=\int_{x_{1}}^{0} \rho_{0}^{-1}(x) d x .
$$

Thus, the boundary condition (3.26) becomes

$$
u=-x_{1} \quad \text { at } y=y_{0}=\int_{x_{1}}^{0} \rho_{0}^{-1}(x) d x
$$


The lower boundary condition becomes, on use of (3.23) and (3.24),

$$
u=0 \quad \text { at } y=0 \text {. }
$$

Finally, the reciprocal relation (3.23) provides the initial condition $\left.u\right|_{\tau=0}$ implicitly via

$$
y=\int_{x_{1}}^{\left.u\right|_{r=0}+x_{1}} \rho_{0}^{-1}(x) d x .
$$

On application of the Hopf-Cole transformation $[8,13]$

$$
u=\phi_{y} / \phi
$$

We obtain the linear boundary-value problem

$$
\begin{array}{rlrl}
\phi_{\tau} & =\phi_{y y}, \\
\phi_{y}+x_{1} \phi & =0 & & \text { at } y=y_{0}, \\
\phi_{y} & =0 & & \text { at } y=0
\end{array}
$$

together with initial condition (3.29). In certain cases the latter can be inverted to give

$$
u(y, 0)=u_{0}(y) .
$$

For example, given uniform initial water content

$$
\theta=\theta_{0}=\text { constant } \quad \text { at } t=0
$$

(3.19) gives

$$
\rho_{0}(x)=\frac{2 a}{q} \frac{(1-m x)}{\left(\theta_{0}-b\right)}
$$

so that, on use of (3.10) and (3.29),

$$
u_{0}(y)=m^{-1} \exp (-m \ell)\left[1-\exp \left(\frac{2 a m y}{\left(b-\theta_{0}\right) q}\right)\right] .
$$

In terms of $\phi$, the initial condition (3.34) gives

$$
\phi=\phi_{0}(y)=\exp \int_{y_{1}}^{y} u_{0}(\sigma) d \sigma
$$

where it is noted that $u=\phi_{y} / \phi$ is independent of the choice of the reference value $y_{1}$.

The linear boundary-value problem (3.30)-(3.32) with initial data (3.35) admits a standard Fourier series solution [7]. Once $\phi(y, \tau)$ has been obtained, $u(y, \tau)$ is given by the relation (3.30) and the solution $\rho(x, s)$ of the intermediate boundary-value problem (3.14)-(3.17) is given parametrically by

$$
\begin{gathered}
\rho=\frac{\partial u}{\partial y}(y, s), \\
x=x_{1}+u(y, s) .
\end{gathered}
$$


The solution $\theta(z, t)$ of the original nonlinear boundary-value problem is likewise given parametrically by

$$
\begin{aligned}
& \theta=b+\frac{2 a}{q}\left(1-m\left[x_{1}+u\left(y, q^{2} t / 4 a\right)\right]\right) / u_{y}\left(y, q^{2} t / 4 a\right), \\
& z=-m^{-1} \ln \left(1-m\left[x_{1}+u\left(y, q^{2} t / 4 a\right)\right]\right) .
\end{aligned}
$$

\section{Steady-state solution for unsaturated flow with extraction}

If the water supplied at the boundary of the finite soil column exactly compensates for the water extraction, then the total water content will remain constant and a steady state will be approached asymptotically at large times. Unlike the transient water distribution, the steady state can be derived explicitly. Thus, the steady-state version of the nonlinear model (3.2)-(3.4) yields

$$
m a(b-\theta)^{-1}-a(b-\theta)^{-2} \theta_{z}-\frac{q}{m} e^{-m z}=\bar{R}-\frac{q}{m} .
$$

In view of the balanced water supply $q x_{1}=\bar{R}$, both boundary conditions are satisfied by $(4.1)$. In terms of the variables $(2.6 \mathrm{a}, \mathrm{b})$,

$$
a(b-w)^{-2} w_{z}=\frac{q}{m} e^{-m z}\left(e^{-m \ell}-1\right)
$$

whence, on integration, we obtain the steady-state water content profile via

$$
b-\theta=(b-w) e^{-m z}=\frac{a e^{-m z}}{\frac{1}{2} q m^{-2}\left[1-e^{-m z}\right]^{2}-\bar{R} m^{-1}\left[1-e^{-m z}\right]+\beta}
$$

where

$$
\beta=\frac{a}{b-\theta(0)}
$$

The latter parameter $\beta$ is related to the total water content invariant $I$ via

$$
I=\int_{0}^{z}(b-\theta) d z=\frac{2 a m}{q} \int_{0}^{1-\exp (-m \zeta)} \frac{d r}{r^{2}-2 \bar{R} m r / q+2 \beta m^{2} / q} .
$$

\section{Acknowledgements}

The first author is grateful for the hospitality of the Department of Mathematics, University of Arizona where this work was written. C. R. acknowledges with gratitude support by the SERC (U.K.). 


\section{References}

[1] G. Bluman and S. Kumei, “On the remarkable nonlinear diffusion equation $(\partial / \partial x)[a(u+$ $\left.b)^{-2}(\partial u / \partial x)\right]-(\partial u / \partial t)=0 ", J$. Math. Phys. 21 (1980) 1019-1023.

[2] N. F. Britton, Reaction-diffusion equations and their applications to biology (Academic Press, London, 1986).

[3] P. Broadbridge, "Selection of solvable nonlinear evolution equations by systematic searches for Lie-Bäcklund symmetries", in Nonlinear evolution equations and dynamical system, (eds. S. Carillo and O. Ragnisco, Lange and Springer, Berlin, 1990).

[4] P. Broadbridge, "Integrable forms of the one-dimensional flow equation for unsaturated heterogeneous porous media", J. Math. Phys. 29 (1988) 622-627.

[5] P. Broadbridge and S. E. Godfrey, "Exact decaying soliton solutions of nonlinear Schrödinger equations: Lie-Bäcklund symmetries", J. Math. Phys. 32 (1991) 8-18.

[6] P. Broadbridge and I. White, "Constant rate rainfall infiltration: a versatile nonlinear model. 1. Analytic solution", Water Resour. Res. 24 (1988) 145-154.

[7] H. S. Carslaw and J. C. Jaeger, Conduction of heat in solids, (Clarendon Press, Oxford, 1959).

[8] J. D. Cole, “One a quasilinear parabolic equation occurring in aerodynamics", Q. Appl. Math. 9 (1951) 225-236.

[9] A. S. Fokas and Y. C. Yortsos, "On the exactly solvable equation occurring in two-phase flow in porous media", SIAM J. Appl. Math 42 (1982) 318-332.

[10] N. C. Freeman and J. Satsuma, "Exact solutions describing an interaction of pulses with compact support in a nonlinear diffusive system”, Phys. Lett. 138 (1989) 110-112.

[11] V. A. Galaktionov, V. A. Dorodnitsyn, G. G. Elenin, S. P. Kurdyumov and A. A. Samarskii, "A quasilinear heat equation with a source: peaking, localization, symmetry exact solutions, asymptotics, structures", J. Soviet Math. 41 (1988) 1222-1292.

[12] A. C. Hearn (ed.), REDUCE user's manual (Rand Corp, Santa Monica, California, 1987).

[13] E. Hopf, "The partial differential equation $u_{t}+u u_{x}=\mu u_{x x}$ ", Comm. Pure Appl. Math. 33 (1950) 201-230.

[14] N. H. Ibragimov, Transformation groups applied to mathematical physics (D. Reidel, Boston, 1985).

[15] J. H. Knight and J. R. Philip, "Exact solutions in nonlinear diffusion", J. Eng. Math. 8 (1974) 219-227.

[16] B. Konopelchenko and C. Rogers, in Nonlinear equations in applied science (Academic Press, New York, to appear, 1991).

[17] D. O. Lomen and A. W. Warrick, "Solution of the one-dimensional linear moisture flow equation with implicit water extraction functions", Soil Sci. Soc. Am. J. 40 (1976) 342-344.

[18] G. S. Ludford, Combustion and chemical reactions (Amer. Math. Soc., Providence, Rhode Island, 1986).

[19] F. J. Molz and I. Remson, "Extraction term models of soil moisture use by transpiring plants", Water Resour. Res. 6 (1970) 1346-1356.

[20] P. J. Olver, Application of Lie groups of differential equations (Springer, New York, 1988).

[21] J. R. Philip, “Theory of infiltration”, Adv. Hydrosciences 5 (1969) 21 5-296.

[22] C. Rogers, “Application of a reciprocal transformation to a two-phase Stefan problem", J. Phys. A: Mathematical and General 18 (1985) 105-109.

[23] C. Rogers, "On a class of moving boundary problems in non-linear heat conduction: application of a Bäcklund transformation", Int. J. Nonlinear Mech 21 (1986) 249-256.

[24] C. Rogers and W. F. Ames, Nonlinear boundary value problems in science and engineering (Academic Press, New York, 1989). 
[25] C. Rogers and P. Broadbridge, "On a nonlinear moving boundary problem with heterogeneity: application of a Bäcklund transformation”, Zeit. Angew. Math. Phys. 39 (1988) 122-128.

[26] C. Rogers, M. P. Stallybrass and D. L. Clements, “On two phase filtration under gravity and with boundary infiltration: application of a Bäcklund transformation", J. Nonlinear Analysis, Theory, Methods and Applications 7 (1983) 785-799.

[27] C. Rogers and P. Wong, "On reciprocal Bäcklund transformations of inverse scattering schemes" Physica Scripta 30 (1984) 10-14.

[28] C. W. Rose and W. R. Stern, "Determination of withdrawal of water from the soil by crop roots as a function of depth and time", Austral. J. Soil Res. 5 (1967) 11-19.

[29] G. Rosen, "Method for the exact solution of a nonlinear diffusion-convection equation", Phys. Lett. 49 (1982) 1844-1846.

[30] G. C. Sander, J.-Y. Parlange, V. Kühnel, W. L. Hogarth, D. Lockington and J. P. J. O'Kane, "Exact nonlinear solution for constant flux infiltration", J. Hydrol. 97 (1988) 341-346.

[31] S. I. Svinolupov, "Second order evolution equations with symmetries", Usp. Mat. Nauka 40 (1985) 241-242.

[32] M. L. Storm, "Heat conduction in simple metals", J. Appl. Phys. 22 (1951) 940-951.

[33] T. Talsma and E. A. Gardner, "Soil water extraction by a mixed eucalypt forest during a drought period", Austr. J. Soil Res. 24 (1986) 25-32.

[34] I. White and P. Broadbridge, "Constant-rate rainfall infiltration: a versatile nonlinear model 2. Applications of solutions", Water Resour. Res. 24 (1988) 155-162.

[35] I. White and M. J. Sully, "Macroscopic and microscopic capillary length and time scales from field infiltration", Water Resour. Res. 23 (1987) 1514-1522.

\section{Appendix \\ The Search for Lie-Bācklund Symmetries}

We assume that

$$
\partial_{t} \mu=\Delta(\mu) \mu_{x x}+R(x, t, \mu)
$$

has a symmetry of the form (2.1). Thus, for any solution $\mu(x, t)$ of (A1), $\mu^{*}(x, t)$ must satisfy

$$
\frac{D \mu^{*}}{D T}=\Delta\left(\mu^{*}\right)\left(\frac{D}{D x}\right)^{2} \mu^{*}+R\left(x, t, \mu^{*}\right)+O\left(s^{2}\right)
$$

where

$$
\frac{D}{D x} F\left(x, t, \mu, \mu_{1}, \mu_{2}, \ldots, \mu_{n}\right):=\frac{\partial F}{\partial x}+\sum_{j=0}^{n} \mu_{j+1} \frac{\partial F}{\partial \mu_{j}}
$$

and

$$
\frac{D F}{D t}=\frac{\partial F}{\partial t}+\sum_{j=0}^{n} \frac{\partial F}{\partial \mu^{j}}\left(\frac{D}{D x}\right)^{j}[K(\mu)]
$$

whenever $\mu$ is a solution of the evolution equation $\partial_{t} \mu=K(\mu)$ under consideration, in this case (A1). 
On substitution of (A1) into (A2), we obtain the set of determining relations for the symmetry generator $L\left(x, t, \mu, \mu_{1}, \mu_{2}, \mu_{3}\right)$. The determining relations for $L$ are contained in a single equation which may, at first, be viewed as a polynomial equation in $\mu_{4}$. The expected highest-order $\mu_{5}$ terms (order 2 for the governing equation plus order 3 for the symmetry) already cancel, by virtue of a general result of Olver [19]. A balance of the $\mu_{4}^{2}$ terms now yields

$$
\frac{\partial^{2} L}{\partial \mu_{3}^{2}}=0 \Rightarrow L=P\left(x, t, \mu, \mu_{1}, \mu_{2}\right)+Q\left(x, t, \mu, \mu_{1}, \mu_{2}\right) \mu_{3}
$$

for functions $P, Q$ to be determined.

Newly derived information on $L$, such as (A5) may be successively fed back into the determining relations. We have used the computer algebra package REDUCE to accomplish this [11]. A balance of the remaining first degree $\mu_{4}$ terms in (A2) now gives

$$
Q=A(t)[\Delta(\mu)]^{3 / 2}
$$

while balance of $\mu_{3}^{2}$ terms implies

$$
P=B\left(x, t, \mu, \mu_{1}\right) \mu_{2}+C\left(x, t, \mu, \mu_{1}\right)
$$

for some functions $B$ and $C$. A balance of $\mu_{3} \mu_{2}$ terms implies that

$$
B=\frac{3}{2} \Delta^{\prime}(\mu) \Delta^{1 / 2} A(t) \mu_{1}+G(x, t, \mu)
$$

for some function $G$. Now, a balance of $\mu_{3} \mu_{1}^{2}$ terms yields

$$
-2 \Delta^{\prime \prime}(\mu) \Delta+\left[\Delta^{\prime}(\mu)\right]^{2}=0
$$

whence

$$
\Delta(\mu)=(\alpha \mu+\beta)^{2}
$$

where $\alpha, \beta$ are constants. Up to this point, our results agree with those of Bluman and Kumei [1] for the reaction-free case $R=0$. However, we must proceed further to derive consistency relations for the reaction term $R(x, t, \mu)$.

For simplictiy, we assume $\Delta(\mu)=\mu^{2}$, since this can always be achieved from (A10) by a linear change of variable. A balance of remaining $\mu_{3}$ terms in (A2) implies that

$$
G=h(x, t) \mu^{2}
$$

and

$$
R=-\frac{1}{3} \frac{A^{\prime}}{A} \mu+\frac{2}{3 A} \frac{\partial h}{\partial x} \mu^{2}
$$


for some function $h$. A balance of $\mu_{2}^{2}$ terms implies that $C$ in (A7) is linear in $\mu_{1}$, so that

$$
C=J(x, t, \mu) \mu_{1}+K(x, t, \mu),
$$

where balance of the remaining $\mu_{2}$ terms shows that

$$
\begin{aligned}
& J=2 \frac{\partial h}{\partial x} \mu^{2}+N(x, t), \\
& K=-\frac{1}{3} \frac{A^{\prime}}{A} h \mu+\frac{1}{2} \frac{\partial h}{\partial t} \mu+\frac{1}{2} \frac{\partial^{2} h}{\partial x^{2}} \mu^{3}+\frac{2}{3 A} \frac{\partial h}{\partial x} h \mu^{2}-\frac{\partial N}{\partial x} \mu,
\end{aligned}
$$

for some function $N$. On balance of $\mu_{1}^{2}$, we now find

$$
h=W(t) x+Y(t)
$$

for functions $W, Y$ to be determined. By equating the coefficients of $\mu_{1} \mu^{2}$ terms to zero, we deduce

$$
N=\left[\frac{1}{3} \frac{A^{\prime}}{A} W-\frac{1}{2} W^{\prime}\right] x^{2}+\alpha_{1}(t) x+\beta_{1}(t)
$$

for some functions $\alpha_{1}$ and $\beta_{1}$. It is now seen that the $\mu_{1} \mu$ terms are already balanced in (A2). This indicates that the determining equations may have a nontrivial solution.

A balance of $\mu_{1}$ terms next reveals that $\alpha_{1}$ and $\alpha_{2}$ must be constants and that $W(t)$ satisfies an equation

$$
\frac{3}{2} W^{\prime}(t)=\frac{A^{\prime}(t)}{A(t)} W+\gamma,
$$

where $\gamma$ is constant. A balance of $\mu^{2} x$ terms shows that $\gamma=0$, whence (A18) implies that

$$
W=\omega[A(t)]^{2 / 3},
$$

with $\omega$ an arbitrary constant. Moreover, a balance of $\mu^{2}$ terms gives

$$
Y=-2 \alpha_{1} A(t)^{2 / 3} \int_{\lambda}^{t} A^{-2 / 3}(\tau) d \tau
$$

where $\lambda$ is an arbitrary constant.

It turns out that the remaining determining relations are already satisfied. Hence, there exists a third-order symmetry of the nonlinear equation

$$
\partial_{t} \mu=\mu^{2} \mu_{x x}+R(x, t, \mu)
$$

provided

$$
R=f(t) \mu^{2}+\mu f^{\prime}(t) / f(t)
$$


where $f(t)=\frac{2}{3} \omega A^{-1 / 3}(t)$. For example, for the Freeman-Satsuma equation which corresponds to $f=2$ in (A22), we may choose $\omega=3$ and $A=1$. Then one symmetry operator is the prolongation of $L \partial / \partial \mu$ with

$$
L=\mu_{3} \mu^{3}=\mu_{2}\left[3 \mu^{2} \mu_{1}+3 x \mu^{2}\right]+6 \mu^{2} \mu_{1}+6 x \mu^{2} .
$$

It is noted that, on a linear change of variables, (A20) generalises to the nonlinear equation (2.3), namely

$$
\partial_{t} \mu=(\alpha \mu+\beta)^{2} \mu_{x x}+(\alpha \mu+\beta)^{2} f(t) / \alpha^{2}+(\alpha \mu+\beta) f^{\prime}(t) /(\alpha f(t)) .
$$

